\title{
Photonic Generation of Frequency 16-Tupling Millimeter-Wave Signal without Optical Filter
}

\author{
Xuan Li1, Shanghong Zhao1, Guodong Wang1, Jingshang Liu² \\ ${ }^{1}$ Information and Navigation College, Air Force Engineering University, Xi'an, China \\ ${ }^{2}$ Troops with No. 93147, Chongqing, China \\ Email: lixuanrch@163.com
}

How to cite this paper: $\mathrm{Li}, \mathrm{X} .$, Zhao, S.H., Wang, G.D. and Liu, J.S. (2021) Photonic Generation of Frequency 16-Tupling Millimeter-Wave Signal without Optical Filter. Journal of Applied Mathematics and Physics, 9, 2995-3005.

https://doi.org/10.4236/jamp.2021.911193

Received: June 9, 2021

Accepted: November 27, 2021

Published: November 30, 2021

\begin{abstract}
A generalized optical filterless approach to achieve photonic generation of frequency 16-tupling millimeter-wave (mm-wave) signal based on two cascaded dual-parallel Mach-Zehnder modulators (DPMZMs) is presented. A theoretical analysis leading to the operating conditions to achieve frequency 16-tupling is developed. Different modulation indices (MIs) can be implemented to achieve the frequency multiplication by adjusting the delay of tunable optical delay line (TODL). It is confirmed by simulation that the proposed scheme is effective, and the radio frequency spurious suppression ratio (RFSSR) of the generated frequency 16-tupling signal can be as high as $40 \mathrm{~dB}$ when the sub-MZMs have extinction ratios of $30 \mathrm{~dB}$. Influencing factors such as extinction ratio, DC bias drift, phase shift deviation and RF voltage deviation on the performance of optical sideband suppression ratio (OSSR) and RFSSR are also investigated.
\end{abstract}

\section{Keywords}

Millimeter-Wave Signal, Frequency 16-Tupling, Dual-Parallel Mach-Zehnder Modulator

\section{Introduction}

Microwave generation by photonic techniques has been intensively investigated for the advantages of low phase noise, high frequency and wide frequency tunability [1] [2]. Many approaches for photonic generation of microwave have been demonstrated in the few past years. Among them, external modulation based on Mach-Zehnder modulator (MZM) for optical frequency multiplication has been considered as an attractive solution due to the system simplicity, operation stability, large frequency tunability and high spectral purity of the generated mi- 
crowave signal [1] [2] [3].

For the external modulation approaches, frequency quadrupling [2]-[8], sextupling [1] [9] and octupling [1] [10] [11] [12] signals have been achieved. To generate higher frequency signal or further reduce the need for the response bandwidth of the modulator, a higher multiplication factor is needed. A frequency 12-tupling scheme was proposed by using a DPMZM, a semiconductor optical amplifier and an optical inter-leaver (IL) [13], the OSSR was $30 \mathrm{~dB}$ for the generated optical signal after the IL, however, the system was complicated, and the IL worked as an OF which limited the utilization for wavelength-division-multiplexing (WDM) systems. A frequency 16-tupling signal can be generated by using two parallel DPMZMs with RFSSR as high as $55 \mathrm{~dB}$ [12], but the MI as 5.520 or 8.654 was too high to realize for a commercial DPMZM. An optical filterless scheme based on three parallel MZMs was proposed to achieve multiplication factors as high as 12 and 18 with RFSSRs as $50 \mathrm{~dB}$ and $32 \mathrm{~dB}$ respectively [14], however, the scheme was hard to practically utilize for the same reason that the MIs should be 5.520 and 9.761 respectively. The two cascaded DPMZMs scheme can be used to generate a frequency 16-tupling signal [15], but no generalized investigation has been reported.

In this paper, a generalized approach to generate 16-tupling mm-wave signal using two cascaded DPMZMs is presented. The significance of the configuration is that different MIs can be implemented to achieve the frequency 16-tupling while the MIs are smaller than 4, which is more practical for utilization. In addition, since no OF is required, the proposal can be used in WDM systems. Optical mm-wave signals with frequencies up to $640 \mathrm{GHz}$ can be achieved by the proposed scheme if two commercially available $40 \mathrm{GHz}$ DPMZMs and a wide enough bandwidth PD are employed.

\section{Principle}

The proposed scheme using two cascaded DPMZMs to achieve frequency 16-tupling mm-wave signal is shown in Figure 1. The system consists of a laser diode (LD), two DPMZMs, a tunable optical delay line (TODL), a photodiode (PD), a local oscillator (LO), an electrical phase shifter (PS), and three electrical power dividers. A low-frequency microwave signal from the LO is divided into

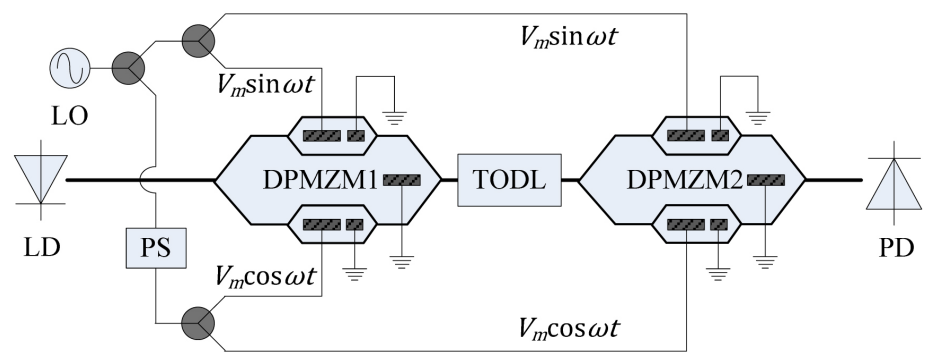

Figure 1. Two cascaded DPMZMs scheme for 16-tupling mm-wave generation. LD, laser diode; LO, local oscillator; PS, phase shifter; TODL, tunable optical delay line; PD, photodiode. 
four paths by three power dividers, and applied to the two DPMZMs. The PS is used after the first power divider to introduce a phase difference between two sub-MZMs of each of the DPMZMs. The TODL can be replaced by two electrical PSs [15]. The function of TODL is to introduce a phase difference between different optical sidebands. Erbium-doped fiber amplifier (EDFA) may be used to boost the optical power of the signals output from DPMZMs.

Assuming that the incident light wave is $E_{0} \cos \left(\omega_{c} t\right)$, where $E_{0}$ is the amplitude of the optical field, and $\omega_{c}$ is the angular frequency of incident light wave. Both the sub-MZMs of DPMZM1 are biased at the maximum transmission point (MATP) to suppress the odd-order sidebands, and the main MZM of DPMZM1 is set to let the signals from the two arms in phase. The phase difference introduced by PS is $\pi / 2$, and then the optical field at the output of the DPMZM1 can be expressed as

$$
E_{1}(t) \approx E_{0} J_{0}(m) \cos \omega_{c} t+E_{0} J_{4}(m)\left[\cos \left(\omega_{c} t-4 \omega t\right)+\cos \left(\omega_{c} t+4 \omega t\right)\right]
$$

where $\omega$ is the angular frequency of the electrical driving signal, $m$ is the MI of DPMZM1, $J_{n}$ is the $n$ th-order Bessel function of the first kind. As can be seen, only the carrier and \pm 4 th-order sidebands are obtained. When the light wave is traveling through the TODL, a phase difference $\varphi$ between the two adjacent sidebands would be introduced and the optical field $E_{T O D}(t)$ at the output of the TODL can be written as [1]

$$
\begin{aligned}
E_{T O D}(t)= & E_{0} J_{0}(m) \cos \left(\omega_{c} t+2 \varphi\right) \\
& +E_{0} J_{4}(m)\left[\cos \left(\omega_{c} t-4 \omega t+\varphi\right)+\cos \left(\omega_{c} t+4 \omega t+3 \varphi\right)\right]
\end{aligned}
$$

$E_{T O D}(t)$ is then sent into DPMZM2. The DPMZM2 is biased identical to the case of DPMZM1, and the MI of DPMZM2 is also $m$. The optical field at the output of DPMZM2 can be expressed as

$$
\begin{aligned}
E_{2}(t)= & E_{0}\left\{J_{0}^{2}(m) \cos \left(\omega_{c} t+2 \varphi\right)+J_{0}(m) J_{4}(m)\left[\cos \left(\omega_{c} t-4 \omega t+2 \varphi\right)\right.\right. \\
& \left.+\cos \left(\omega_{c} t+4 \omega t+2 \varphi\right)\right]+J_{0}(m) J_{4}(m) \cos \left(\omega_{c} t-4 \omega t+\varphi\right) \\
& +J_{4}^{2}(m)\left[\cos \left(\omega_{c} t-8 \omega t+2 \varphi\right)+\cos \left(\omega_{c} t+\varphi\right)\right] \\
& +J_{0}(m) J_{4}(m) \cos \left(\omega_{c} t+4 \omega t+3 \varphi\right) \\
& \left.+J_{4}^{2}(m)\left[\cos \left(\omega_{c} t+3 \varphi\right)+\cos \left(\omega_{c} t+8 \omega t+3 \varphi\right)\right]\right\}
\end{aligned}
$$

If the following conditions are satisfied

$$
\left\{\begin{array} { l } 
{ \varphi = ( 2 k + 1 ) \pi / 2 } \\
{ J _ { 0 } ( m ) = 0 }
\end{array} \text { or } \left\{\begin{array}{l}
\varphi=(2 k+1) \pi \\
J_{0}^{2}(m)=2 J_{4}^{2}(m)
\end{array}\right.\right.
$$

where $k$ is an integer, $E_{2}(t)$ can be simplified into

$$
E_{2}(t)=E_{0}\left\{J_{4}^{2}(m)\left[\cos \left(\omega_{c} t-8 \omega t+2 \varphi\right)+\cos \left(\omega_{c} t+8 \omega t+3 \varphi\right)\right]\right\}
$$

As can be seen, only the \pm 8 th-order sidebands are presented, the beating of the two sidebands at the PD would generate a frequency 16-tupling mm-wave signal. 
Figure 2 schematically depicts the principle of the proposed mm-wave generation scheme. Optical spectra with order of $4 n$ sidebands are performed for both of DPMZM1 and DPMZM2. There are two methods to achieve a frequency 16-tupling signal with the proposed scheme. For the first method, as shown in the upper part of Figure 2, the MI is set to make the carrier be suppressed, and only \pm 4 th-order sidebands are presented at the output of DPMZM1. An additional phase delay $\pi$ between the two components is introduced by TODL. At the output of DPMZM2, the \pm 4 th-order sidebands are suppressed as the MI is set to make the value of $J_{0}(m)$ equals to 0 , and the two optical components with the same frequency as the original incident light wave are also suppressed as they have identical amplitudes and opposite phases. For the other method, as shown in the lower part of Figure 2, the carrier is not suppressed at the output DPMZM1 and three components are presented. A phase difference $\pi$ between the carrier and \pm 4 th-order sidebands is introduced by the TODL. At the output of DPMZM2, the two +4th-order sidebands, two -4 th-order sidebands and the three carrier sidebands are vanished respectively, for they have identical amplitudes and opposite phase. Thus, only the \pm 8 th-order sidebands are presented at the output of the system.

\section{Simulation Results and Discussion}

A concept-proof of frequency 16-tupling optical mm-wave generation is built based on the OptiSystem to verify the proposed scheme. The laser frequency is 193.1 THz with an output power of $10 \mathrm{dBm}$, the LO frequency is $10 \mathrm{GHz}$, the insert loss of DPMZM is $5 \mathrm{~dB}$, the extinction ratios of sub-MZMs are $30 \mathrm{~dB}$, the responsivity of $\mathrm{PD}$ is $0.65 \mathrm{~A} / \mathrm{W}$.

Firstly, the conditions presented in equation (4) are calculated. When $0<m<$ 5 , the value of $J_{0}(m)$ will be equal to 0 when $m=2.405$, and the condition $J_{0}^{2}(m)$ $=2 J_{4}^{2}(m)$ will be satisfied when $m=2.265,2.672$ or 3.999. Therefore, there are four MIs can be used for the proposed approach to generate a 16-tupling mm-wave signal.

The MI is set as 2.405 to suppress the carrier, and the time delay of the TODL is set as $11 / 160 \mathrm{~ns}$, an EDFA with gain of $15 \mathrm{~dB}$ and noise figure of $4 \mathrm{~dB}$ is placed

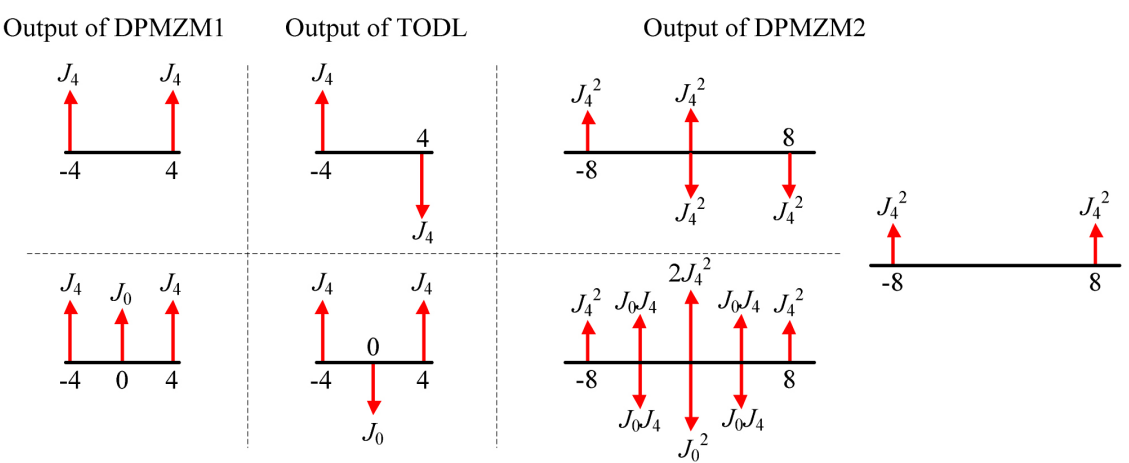

Figure 2. Illustration for the proposed mm-wave generation. 
after DPMZM1, and another EDFA with gain of $25 \mathrm{~dB}$ and noise figure of $4 \mathrm{~dB}$ is placed after DPMZM2, the average receiving optical signal power of PD appropriates $-4 \mathrm{dBm}$. Simulation results are given in Figure 3. The dashed line in Figure 3(a) represent the power spectral density of amplifier spontaneous emission (ASE) noise induced by EDFAs. It can be seen that a $160 \mathrm{GHz} \mathrm{mm}$-wave signal is generated by a $10 \mathrm{GHz}$ oscillator. Several optical sidebands are presented and the fifth-order sidebands become the second dominant components. In the electrical domain, the RFSSR is limited by the tenth-order harmonic component. The measured OSSR and RFSSR of the generated mm-wave signal are degraded to $9 \mathrm{~dB}$ and $18 \mathrm{~dB}$ respectively, as shown in Figure 3(a) and Figure 3(b).

The MI is set as 2.265, the time delay of the TODL is set as 11/80 ns, an EDFA with gain of $15 \mathrm{~dB}$ and noise figure of $4 \mathrm{~dB}$ is placed after DPMZM1, and another EDFA with gain of $29 \mathrm{~dB}$ and noise figure of $4 \mathrm{~dB}$ is placed after DPMZM2, the average receiving optical signal power of PD appropriates $-4 \mathrm{dBm}$. Simulation results are given in Figure 4. For the generated optical signal, many components are presented and the OSSR is only $4 \mathrm{~dB}$. In the electrical domain, the even-order harmonic components are appeared and the RFSSR only

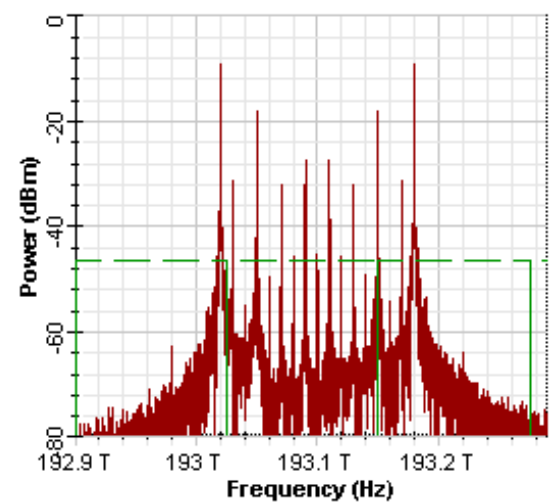

(a)

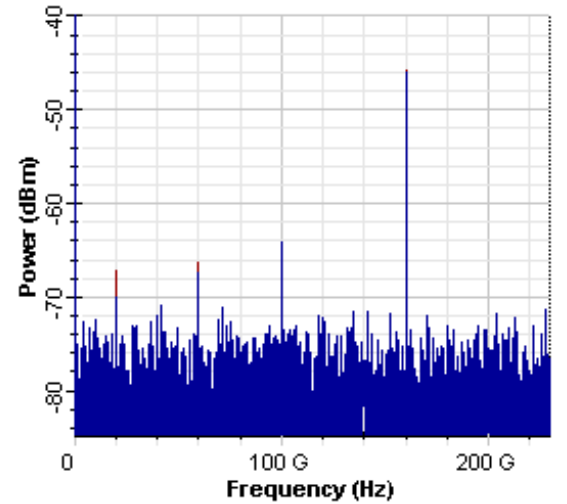

(b)

Figure 3. (a) Optical, (b) electrical spectrum when $\mathrm{m}=2.405$.

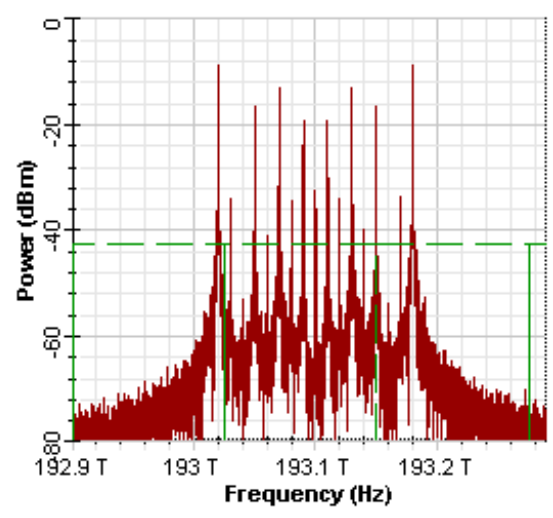

(a)

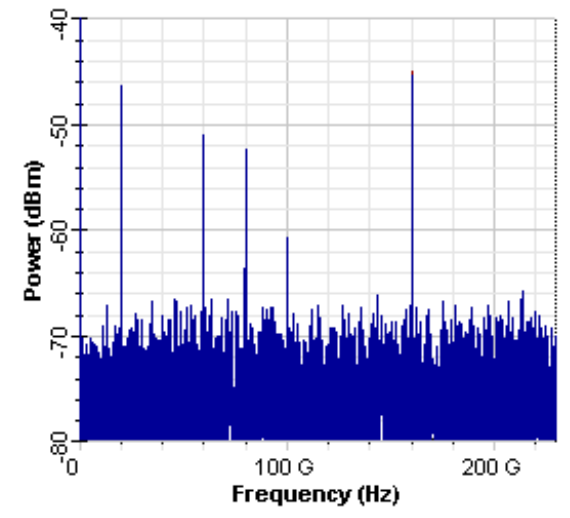

(b)

Figure 4. (a) Optical, (b) electrical spectrum when $\mathrm{m}=2.265$. 
appropriates $1 \mathrm{~dB}$. To obtain the generated $160 \mathrm{GHz} \mathrm{mm}$-wave signal, an electrical filter is needed.

The MI is set as 2.672, the time delay of the TODL is set as 11/80 ns, an EDFA with gain of $15 \mathrm{~dB}$ and noise figure of $4 \mathrm{~dB}$ is placed after DPMZM1, and another EDFA with gain of $19 \mathrm{~dB}$ and noise figure of $4 \mathrm{~dB}$ is placed after DPMZM2, the average receiving optical signal power of PD appropriates $-4 \mathrm{dBm}$. Simulation results are given in Figure 5. The fifth-order sidebands become the second dominant components in the optical domain and the OSSR is $13 \mathrm{~dB}$. For the generated electrical signal, the RFSSR is limited by the sixth-order harmonic component and it is about $26 \mathrm{~dB}$.

The MI is set as 3.999 , the time delay of the TODL is still set as $11 / 80 \mathrm{~ns}$. As the optical power output from DPMZM1 is high, thus just one EDFA is needed and placed after the DPMZM2, the gain of the EDFA is $15 \mathrm{~dB}$ and the noise figure is $4 \mathrm{~dB}$. The average receiving optical signal power of $\mathrm{PD}$ appropriates -4 $\mathrm{dBm}$. Simulation results are given in Figure 6. The third-order sidebands become the second dominant components in the optical domain, and the OSSR is $20 \mathrm{~dB}$. For the generated electrical signal, the RFSSR is limited by the sixth-order harmonic and it is about $40 \mathrm{~dB}$.

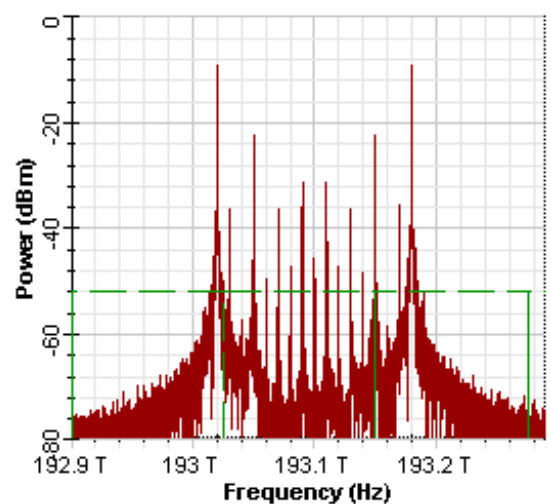

(a)

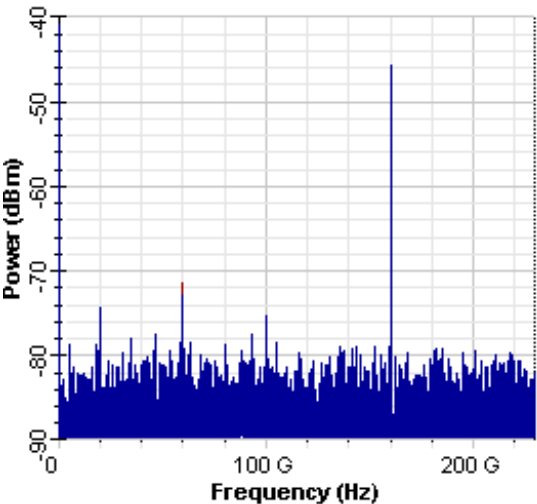

(b)

Figure 5. (a) Optical, (b) electrical spectrum when $\mathrm{m}=2.672$.

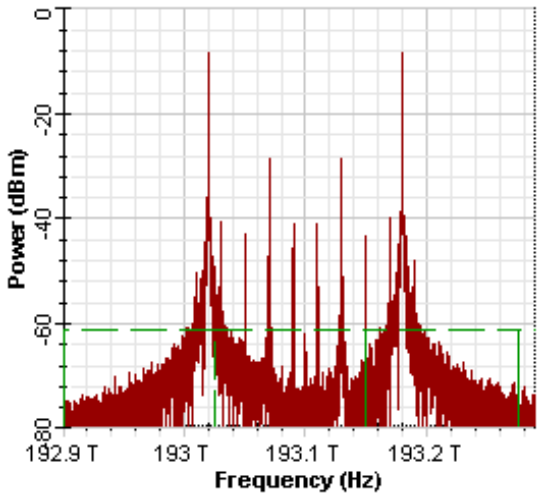

(a)

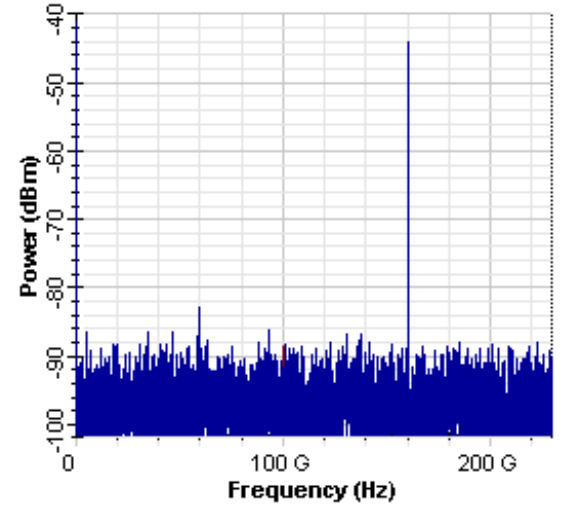

(b)

Figure 6. (a) Optical, (b) electrical spectrum when $\mathrm{m}=3.999$. 
It can be seen from Figures 3-6 that the simulation results agree well with the theoretical analysis. Frequency 16-tupling mm-wave signals are generated with RFSSRs about $40 \mathrm{~dB}, 26 \mathrm{~dB}$ and $19 \mathrm{~dB}$ when $m=3.999,2.672$ and 2.405 respectively.

The generation of the mm-wave signal is achieved by adjusting the voltage of the oscillator, the phase difference between the driving signal, the time delay of the TODL, and the DC bias of the modulators. For the practical application, these parameters are not ideal, and they may degrade the performance of the proposed scheme. The influence of imperfect factors on the performance of OSSR and RFSSR for the generated mm-wave signal will be discussed in this part.

Figure 7 shows the simulation results of the OSSR and RFSSR against the power split ratio of the sub-MZM. The range of the extinction ratio of sub-MZM is $20-50 \mathrm{~dB}$. The OSSR has a linear relationship with the extinction ratio as shown in Figure 7(a). The RFSSR increases along with the increase of extinction ratio firstly, because the undesired components are suppressed better when the extinction ratio is higher. Then the RFSSR retains unchanged when the extinction ratio is high enough, because the undesired components are lower than the noise power and the RFSSR is restricted by the noise. It also can be seen that, when the extinction ratio is given, a better performance can be obtained when a higher MI is provided.

Figure 8 shows the simulation results of the OSSR and RFSSR against the bias drift of the sub-MZMs when the extinction ratios are $30 \mathrm{~dB}$. The bias voltage deviation ratio is defined as $\left(\Delta V / V_{\pi}\right) \times 100 \%$, where $\Delta V$ is bias voltage deviation. About $3 \mathrm{~dB}$ OSSR degradations are observed with 2\% bias drift. The RFSSRs are more sensitive to the bias drift. When $m=3.999$, a degradation of $26 \mathrm{~dB}$ is observed with $2 \%$ bias drift, but the RFSSR can be higher than $17 \mathrm{~dB}$ when the bias voltage deviation ratio is controlled within $\pm 2 \%$ using bias feedback control circuit.

Figure 9 shows the simulation results of the OSSR and RFSSR against the imperfect phase delay of the PS with varying phase deviation from -2 to 2 degree.

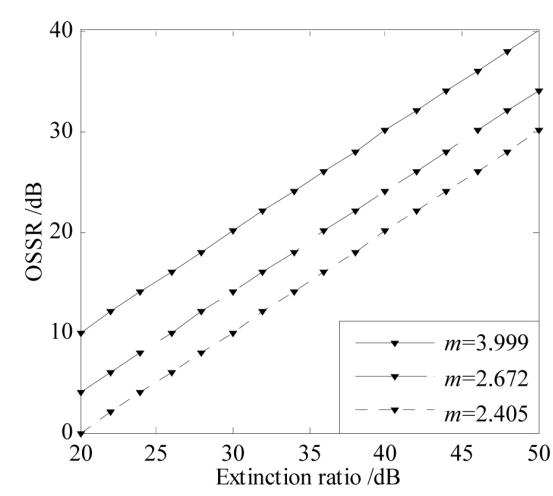

(a)

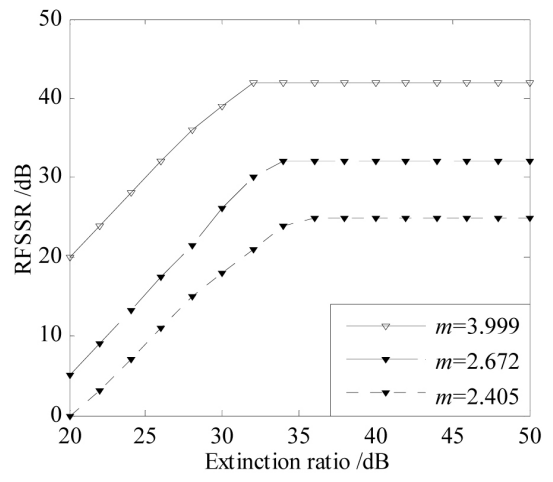

(b)

Figure 7. (a) OSSR, (b) RFSSR versus extinction ratio. 


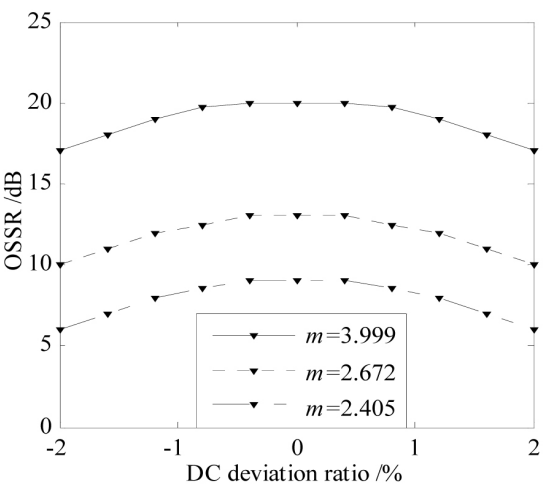

(a)

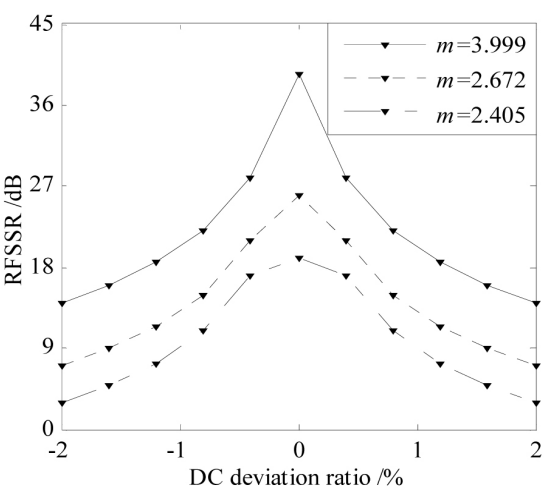

(b)

Figure 8. (a) OSSR, (b) RFSSR versus bias deviation.

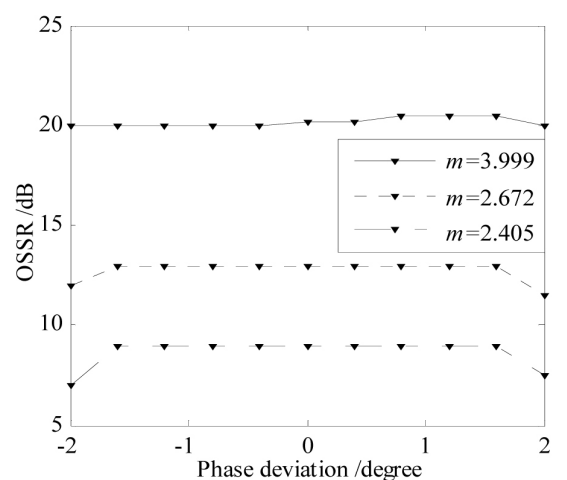

(a)

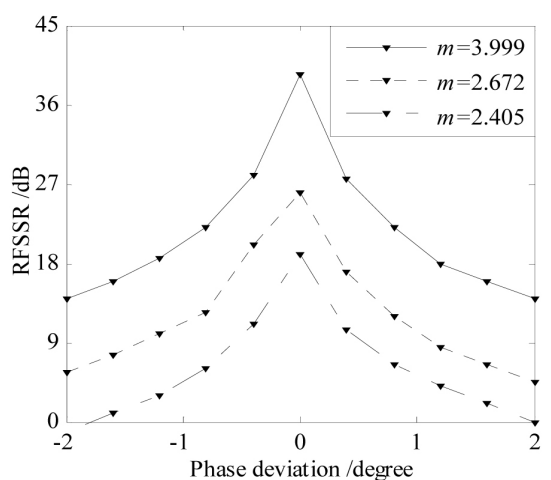

(b)

Figure 9. (a) OSSR, (b) RFSSR versus phase deviation of PS.

If the phase deviation is smaller than \pm 1.5 degree, there are no significant degradations for OSSRs. Note that the maximal OSSR with $m=3.999$ does not correspond to the desired phase difference, because the OSSR is not only depended on the phase difference, but also the extinction ratio. In the electrical domain, the phase deviation obviously degrades the RFSSR. When the phase deviation ranges from -2 to 2 degree, the RFSSRs degrade form $40 \mathrm{~dB}$ to $14 \mathrm{~dB}$, from $26 \mathrm{~dB}$ to $6 \mathrm{~dB}$, and from $19 \mathrm{~dB}$ to zero when $m=3.999,2.672$ and 2.405 respectively.

Figure 10 shows the simulation results of the OSSR and RFSSR against the RF driving voltage deviation when the extinction ratios of sub-MZMs are $30 \mathrm{~dB}$. The normalized RF voltage deviation is defined as $\left(\Delta V_{p p} / V_{p p}\right) \times 100 \%$, where $\Delta V_{p p}$ is the RF driving voltage deviation. If the normalized RF voltage deviation is smaller than $\pm 1 \%$, there are no significant degradations for OSSRs, but degradations of $26 \mathrm{~dB}, 20 \mathrm{~dB}$ and $14 \mathrm{~dB}$ are observed for RFSSRs with $m=3.999$, 2.672 and 2.405 respectively.

Finally, we give a comprehensive comparison of the four conditions that are used to generate a 16-tupling mm-wave signal. Assuming that the half-wave voltage of sub-MZM is $4.5 \mathrm{~V}$, then the microwave power of LO for different MIs can be calculated as shown in the Table 1 . The power penalty of optical system 


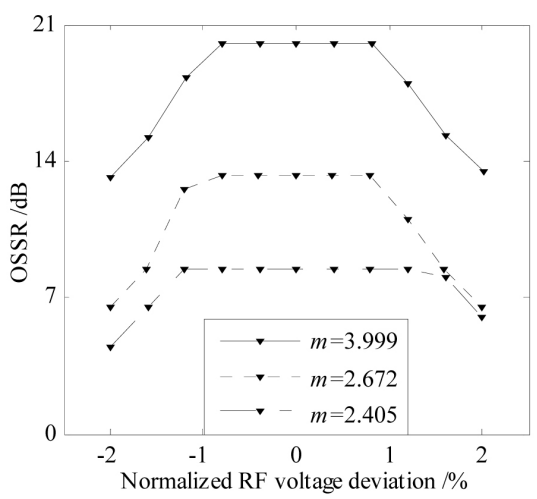

(a)

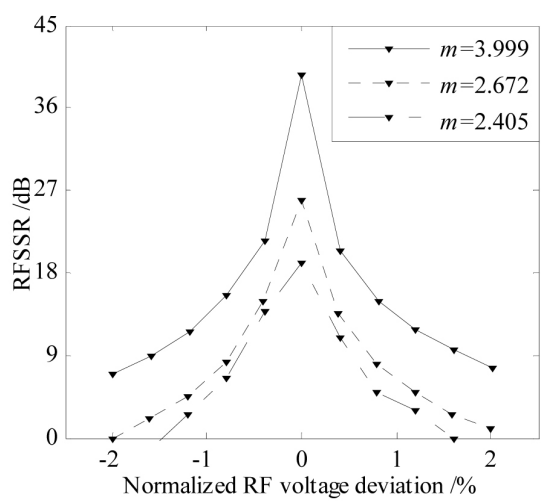

(b)

Figure 10. (a) OSSR, (b) RFSSR versus RF voltage deviation of PS.

Table 1. Power comparison of the four conditions with different MIs.

\begin{tabular}{cccc}
\hline MI & $\begin{array}{c}\text { Microwave power } \\
\text { of LO }\left(V_{\pi}=4.5 \mathrm{~V}\right)\end{array}$ & $\begin{array}{c}\text { Power penalty of } \\
\text { optical system }\end{array}$ & $\begin{array}{c}\text { Power compensation } \\
\text { of optical amplifier }\end{array}$ \\
\hline 3.999 & $37 \mathrm{dBm}$ & $-19 \mathrm{~dB}$ & $15 \mathrm{~dB}$ \\
2.672 & $34 \mathrm{dBm}$ & $-39 \mathrm{~dB}$ & $35 \mathrm{~dB}$ \\
2.405 & $33 \mathrm{dBm}$ & $-44 \mathrm{~dB}$ & $40 \mathrm{~dB}$ \\
2.265 & $32 \mathrm{dBm}$ & $-49 \mathrm{~dB}$ & $45 \mathrm{~dB}$ \\
\hline
\end{tabular}

means that, when no EDFA is used, the optical power loss between the output of DPMZM2 and the output of LD, and they are estimated by the simulation results. The power compensation of optical amplifier means the gains needed in the simulation to guarantee the average receiving optical signal power of PD appropriates $-4 \mathrm{dBm}$. It can be seen that when $m=3.999$, the power of LO is $3 \mathrm{~dB}$ - $5 \mathrm{~dB}$ higher than the other three cases, but the power penalty of optical system is $20 \mathrm{~dB}-30 \mathrm{~dB}$ lower.

\section{Conclusion}

A filterless frequency 16-tupling optical mm-wave signal generation approach is demonstrated. Compared with the reference [15], this paper gives a generalized investigation of the two cascaded DPMZMs scheme. Different MIs can be implemented to achieve the frequency 16-tupling signal by adjusting the delay of TODL. It is confirmed by simulation that the proposed scheme is effective. Influencing factors such as extinction ratio, DC bias drift, PS deviation and RF voltage deviation on the performance of OSSR and RFSSR are investigated. The simulation results show that the RFSSR of the generated frequency 16-tupling signal can be as high as $40 \mathrm{~dB}$ when the sub-MZMs have extinction ratios of 30 dB. Compared with the previous high-frequency multiplication factor schemes proposed in references [13] [15], the cascaded DPMZMs approach is more practical for application, because the value of MI is $2.405,2.672$ or 3.999 , which is much lower than the previous schemes as $5.520,8.654$ or 9.761 . 


\section{Acknowledgements}

This work was supported by the National Natural Science Foundation of China (61901507).

\section{Conflicts of Interest}

The authors declare no conflicts of interest regarding the publication of this paper.

\section{References}

[1] Li, W. and Yao, J. (2010) Investigation of Photonically Assisted Microwave Frequency Multiplication Based on External Modulation. IEEE Transactions on Microwave Theory and Techniques, 58, 3259-3268. https://doi.org/10.1109/TMTT.2010.2075671

[2] Liu, W., Wang, M. and Yao, J. (2013) Tunable Microwave and Sub-Terahertz Generation Based on Frequency Quadrupling Using a Single Polarization Modulator. Journal of Lightwave Technology, 31, 1636-1644. https://doi.org/10.1109/JLT.2013.2254699

[3] Zhou, F., Jin, X., Yang, B., et al. (2013) Photonic Generation of Frequency Quadrupling Signal for Millimeter-Wave Communication. Optics Communications, 304, 71-74. https://doi.org/10.1016/j.optcom.2013.04.030

[4] Zhang, J., Chen, H., Chen, M., et al. (2007) A Photonic Microwave Frequency Quadrupler Using Two Cascaded Intensity Modulators with Repetitious Optical Carrier Suppression. IEEE Photonics Technology Letters, 19, 1057-1059.

https://doi.org/10.1109/LPT.2007.899462

[5] Chi, H. and Yao, J. (2008) Frequency Quadrupling and Upconversion in a Radio Over Fiber Link. Journal of Lightwave Technology, 26, 2706-2711. https://doi.org/10.1109/JLT.2008.927613

[6] Lin, C., Shih, P., Chen, J., et al. (2009) Optical Millimeter-Wave Up-Conversion Employing Frequency Quadrupling without Optical Filtering. IEEE Transactions on Microwave Theory and Techniques, 57, 2084-2092. https://doi.org/10.1109/TMTT.2009.2015036

[7] Laurêncio, P., Vargues, H., Avó, R., et al. (2010) Generation and Transmission of Millimeter Wave Signals Employing Optical Frequency Quadrupling. International Conference on Transparent Optical Networks IEEE. https://doi.org/10.1109/ICTON.2010.5548961

[8] Li, H., Huang, T., et al. (2014) Photonic Generation of Frequency-Quadrupled Microwave Signal with Tunable Phase Shift. IEEE Photonics Technology Letters, 26, 220-223. https://doi.org/10.1109/LPT.2013.2290691

[9] Pan, S.L. and Yao, J.P. (2010) Tunable Subterahertz Wave Generation Based on Photonic Frequency Sextupling Using a Polarization Modulator and a Wavelength-Fixed Notch Filter. IEEE Transactions on Microwave Theory and Techniques, 58, 1967-1975. https://doi.org/10.1109/TMTT.2010.2050182

[10] Zhang, Y. and Pan, S. (2012) Experimental Demonstration of Frequency-Octupled Millimeter-Wave Signal Generation Based on a Dual-Parallel Mach-Zehnder Modulator. 2012 IEEE MTT-S International Microwave Workshop Series on Millimeter Wave Wireless Technology and Applications. https://doi.org/10.1109/IMWS2.2012.6338202

[11] Lin, C., Shih, P., Jiang, W., et al. (2009) A Continuously Tunable and Filterless Opt- 
ical Millimeter-Wave Generation via Frequency Octupling. Optics Express, 17, 19749-19756. https://doi.org/10.1364/OE.17.019749

[12] Yin, X., Wen, A., Chen, Y., et al. (2011) Studies in an Optical Millimeter-Wave Generation Scheme via Two Parallel Dual-Parallel Mach-Zehnder Modulators. Journal of Modern Optics, 58, 665-673.

https://doi.org/10.1080/09500340.2011.565375

[13] Shih, P., Chen, J., Lin, C., et al. (2010) Optical Millimeter-Wave Signal Generation Via Frequency 12-Tupling. Journal of Lightwave Technology, 28, 71-77. https://doi.org/10.1109/JLT.2009.2035452

[14] Chen, Y., Wen, A., Guo, J., et al. (2011) A Novel Optical Mm-Wave Generation Scheme Based on Three Parallel Ma Ch-Zehnder Modulators. Optics Communications, 284, 1159-1169. https://doi.org/10.1016/j.optcom.2010.11.012

[15] Chen, X., Liu, Z., Jiang, C., et al. (2013) A Filterless Optical Millimeter-Wave Generation Based on Frequency 16-Tupling. Asia Communications and Photonics Conference. 